\title{
Association of masseter muscle activities during awake and sleep periods with self-reported anxiety, depression, and somatic symptoms
}

\begin{abstract}
Aim and background: The objective of this study was to determine if duty factors (DF) of low-magnitude MMA during awake and sleep periods were associated with self-reports of anxiety, depression, and somatic symptoms, and if so, whether or not any associations were modified by gender or the presence of pain. Limited information is currently available in the literature regarding the association of low-magnitude masseter muscle activities (MMA) in habitual environmental settings and the presence of psychological symptoms.

Materials and Methods: Sixty-eight consenting participants were classified using the Diagnostic Criteria for Temporomandibular Disorders examination and validated selfreporting psychological symptom evaluation questionnaires. Each subject also had masseter electromyography recordings during standardized biting tasks in 2 laboratory sessions to calibrate the in-field MMA collected during 3 awake and 3 sleep periods.

Results: During awake periods, subjects with self-reported depression and somatic symptoms had statistically high odds of having higher DF of low-magnitude MMA (defined by $\geq 75$ th percentile of sample). The association between high DF of low-magnitude MMA and self-reported depression symptoms was significantly augmented among male participants, whereas, the association between high DF of low-magnitude MMA and selfreported somatic symptoms was significantly increased among female participants without pain.
\end{abstract}

Conclusion: These pilot data support associations of low-magnitude masseter muscle activities with self-reported depression and somatic symptoms during awake periods

Keywords: masticatory muscles, pain, gender, temporomandibular joint disorders, electromyography, psychological factors
Volume 2 Issue I - 2015

\author{
Shehryar N Khawaja,' Laura R Iwasaki, ${ }^{2}$ \\ Robert Dunford, ${ }^{3}$ Jeffrey C Nickel, ${ }^{2}$ Willard \\ McCall,' Heidi C Crow, 'Yoly Gonzalez' \\ 'Oral Diagnostic Sciences, University at Buffalo, USA \\ ${ }^{2}$ Departments of Orthodontics \& Dentofacial Orthopedics and \\ Oral \& Craniofacial Sciences, University of Missouri-Kansas \\ City, USA \\ ${ }^{3}$ Department of Oral Biology, University at Buffalo, USA
}

Correspondence: Shehryar N Khawaja, University at Buffalo, 355 Squire Hall, 3435 Main St. Buffalo, NY I42I4, USA, Email khawajashehryar@gmail.com

Received: February 12, 2015 | Published: March 13, 2015
Abbreviations: MMA, masticatory muscle activities; DF, duty factors; EMG, electromyography; TMD, temporomandibular disorders; DC-TMD, diagnostic criteria for temporomandibular disorders; GAD 7, generalized anxiety disorders 7-item scale; PHQ 9, patient health questionnaire 9; PHQ 15, patient health questionnaire 15; OR, odds-ratios

\section{Introduction}

Functional and non-functional jaw use during awake and sleep periods requires masticatory muscle activation that can be characterized using electromyography (EMG) according to the magnitude and duration of muscle activities expended. ${ }^{1}$ During nonfatiguing situations when applied force directions are consistent, magnitudes of masticatory muscle activities recorded via EMG are linearly related to the occlusal forces produced ${ }^{2}$ and these relations are repeatable. ${ }^{3}$ High duration or frequency of masticatory muscle activity, at any magnitude, is considered to be a risk factor for dental attrition, hypersensitivity of teeth, breakage of restorations, tooth fracture, periodontal diseases, ${ }^{4}$ and temporomandibular disorders (TMD) ${ }^{4-6}$ The etiological concept of masticatory muscle activity has undergone a conceptual shift over time from peripheral (i.e. occlusal) to central (i.e. anxiety) regulation. Studies on jaw use resulting in high masticatory muscle activities have suggested that the presence of psychological factors, such as anxiety, depression, and somatic symptoms may play a role..$^{7-10}$ However, the reported data have been inconsistent. ${ }^{7}$ This inconsistency may be due to variable criteria being used to characterize the activity of masticatory muscles or the presence of psychological symptoms, and inclusion of confounding factors such as gender, age, and the presence of pain. ${ }^{7-9}$ Furthermore, limited research is available regarding the magnitude, duration, and frequency of masticatory muscle activities recorded outside of laboratory settings in peoples' habitual environments. Recent research has indicated that jaw use involving low magnitudes of masticatory muscle activities may be associated with the presence of pain in the orofacial region. ${ }^{11-14}$ This is in contrast to most past observational studies, which have focused on high magnitudes of masticatory muscle activities associated with clenching or bruxing with large occlusal forces. Limited data are available on the etiology of low magnitudes of masticatory muscle activities.

The aims of this pilot study were to determine if masseter muscle activities performed at low magnitudes during awake and sleep periods in participants were associated with self-reports of anxiety, 
depression, and physical symptoms, and if so, whether or not any associations were modified by gender or presence of orofacial pain, such as pain-related TMD. A calibrated ambulatory EMG monitoring system $^{15}$ was used to measure the awake and sleep time-period masseter muscle activities. Validated self-report questionnaires were used to record the presence of psychological symptoms, ${ }^{16-18}$ and a standardized Diagnostic Criteria for Temporomandibular Disorders (DC-TMD) examination protocol was used to classify subjects. ${ }^{19}$

\section{Materials and methods}

\section{Study participants}

The study was approved by the Health Sciences Institutional Review Boards of the State University of New York at Buffalo and University of Missouri-Kansas City. Informed consent was obtained from each participant. All participants were recruited at the University at Buffalo, School of Dental Medicine in response to community advertisements. Participants were healthy males and females with intact dentitions, who were able to read or follow tasks associated with the laboratory and field recordings. Participants, who reported to be pregnant, had any musculoskeletal disease, such as fibromyalgia, muscular atrophy, or arthritis, and who exhibited gross asymmetries in craniomandibular anatomy as determined by examination were excluded.

\section{Study appointments}

A summary of the study appointments is provided in (Figure 1). At the initial visit, an examiner explained the study, obtained informed consent, reviewed the participant's medical history, and performed a screening clinical examination to test inclusion and exclusion criteria. At a subsequent visit, those who met the inclusion criteria underwent the standardized DC-TMD examination protocol and completed the DC/TMD Axis II forms: Generalized Anxiety Disorders 7-item scale (GAD 7), Patient Health Questionnaire-9 (PHQ-9), and Patient Health Questionnaire-15 (PHQ 15). ${ }^{20}$

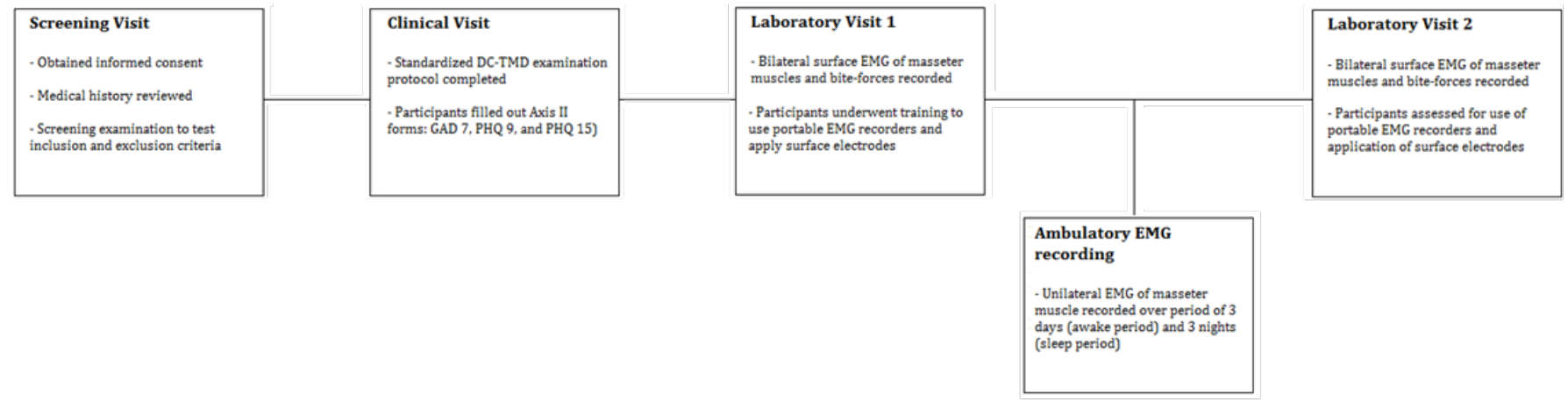

Figure I Summary of the study appointments.

Participants eligible for the study then presented for 2 laboratory sessions during which bilateral surface EMG from masseter muscles and bite-forces were recorded during standardized molar biting tasks on each of their right and left sides. These laboratory data were used to calibrate ambulatory masseter EMG for individual subjects recorded in the field. In addition, during the time between the 2 laboratory visits participants recorded unilateral ambulatory EMG of masseter muscle. In order to make EMG recordings reliable, participants were trained to use portable EMG recorders and surface electrodes on one side of the face during the first laboratory visit. Similarly, in the second laboratory visit, participants underwent assessment for the use of portable EMG recorders and application of surface electrodes. In-field EMG data and equipment were returned at the second laboratory visit.

\section{Equipment and methods}

Laboratory recordings during standardized biting tasks for calibration: For laboratory EMG recordings, bipolar surface electrodes, amplifiers, and a digital recording system were used as previously described. ${ }^{3}$ In brief, the superficial masseter muscles were palpated during tooth-clenching and then, the skin over the main belly of each of the right and left muscles and behind the right ear was cleaned with isopropyl alcohol wipes (Kendall WebcolTM, Tyco Healthcare group LP, Mansfield, MA, USA). Self-adhesive surface electrodes preloaded with conducting gel (Neurology 72000-S, Ambu, Sphereerup, Denmark) were applied in pairs to the masseter muscles' centroids bilaterally and singly, as a ground, over the right mastoid process. EMG signals were amplified (10 P511 AC Grass Preamplifiers ${ }^{\circledR}$, Astro-Med Inc., West Warwick RI), filtered (high pass: $0.03 \mathrm{kHz}$ and low pass: $3.0 \mathrm{kHz}$ ), sampled at 2000 samples/s/channel, and viewed and stored on tape using commercial software (PC Scan MKII PCIF250NI, Sony Magnescale America Inc., Farmington Hill, MI). Custom bite-force transducers (Figure 2) as described previously were used. ${ }^{21}$ In brief, these were made of electrically resistant film (Flexforce, Tekscan Inc., Boston, MA) attached to a stainless steel half-sphere bolus with wire handle and sandwiched by acrylic (Triad, TruTray, Denstply Inc., York, PA), then wrapped with clean, clear plastic film. Each transducer was pre-calibrated so the electrical output $(\mathrm{V})$, recorded simultaneously with the masseter EMG, could be converted into units of force, Newton $(\mathrm{N})$. The same standardized static and dynamic biting tasks were performed by each subject at each of 2 laboratory visits while an investigator held the bite-force transducer between the first molars and EMG and force outputs were recorded. Subjects were instructed to use light to medium bite-forces of their own accord and were not provided visual or other feedback. For each of 5 static bites per side and visit, the subject delivered a relatively steady bite-force for about $3 \mathrm{~s}$ with a rest in between bites. For dynamic bites on each side at each visit, subjects followed a digital metronome signal set at 4 frequencies $(0.5,1,1.5$ and $2.0 \mathrm{~Hz})$ and at each frequency provided at least 5 bites of varied low-medium magnitudes. 


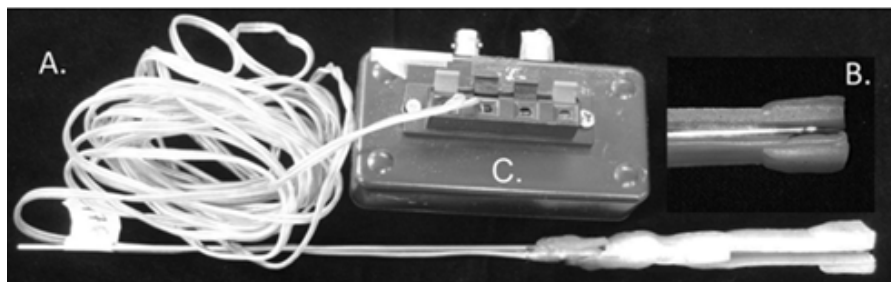

Figure 2 Bite-force transducer.

Figure 2A Bite-force transducer consisting of acrylic biting surfaces sandwiching.

Figure 2B Sensor film (Tekscan Flexiforce ${ }^{\circ}$, Tekscan Inc., South Boston, MA USA) attached to a wire handle. Sensor film is soldered to connector wires, which are plastic-coated and plugged into.

Figure 2C An amplifier, which is then connected to a tape recorder for data collection.

Ambulatory EMG of masseter muscles: For in-field EMG recordings, participants used portable EMG equipment (Figure 3) and supplies as instructed verbally and via an illustrated manual, to capture unilateral masseter muscle activities during both functional and non-functional jaw movements. Subjects chose side of recording, and recorded on the same side for total periods of at least 6hours for each of 3days (awake period) and 3nights (sleep period). Methods for palpating the centroid of the masseter muscle, preparing overlying skin surfaces, and applying in-field EMG surface electrodes (Pre-Gelled Disposable Surface Electrode, Alpine Biomed Corp., Fountain Valley, CA, USA) were the same as used for the laboratory EMG recordings. The in-field surface electrode signals were band pass filtered (20$1000 \mathrm{~Hz})$ and amplified $(5000 \mathrm{x})$ by means of the portable device with the digital amplifier having an input impedance of $250 \mathrm{M} \Omega$, noise level of $0.7 \mu \mathrm{V}$, and common mode rejection ratio of $100 \mathrm{~dB}$. In-field EMG signals were collected using data storage cards (512MB MMC Mobile Multimedia Flash Memory Card, Pretec Electronics Corp., Taipei, Taiwan).

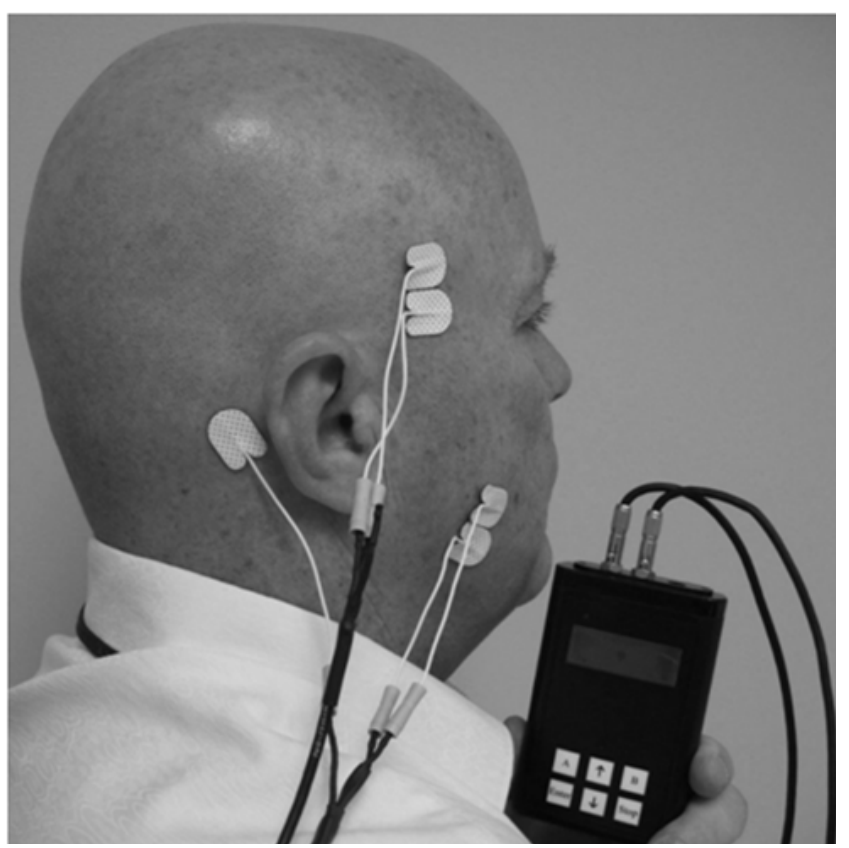

Figure 3 Ambulatory EMG setup.
Picture illustrating ambulatory EMG setup, where self-adhering surface electrodes are affixed over the anterior temporalis muscle, the body of masseter muscle and mastoid process (single ground electrode). Electrodes are connected to a portable EMG device.

\section{Data processing}

Masseter muscle EMG calibration: Similar to previously applied approaches ${ }^{15}$ where, using a first approximation of symmetry, right masseter EMG activities versus bite forces recorded for unilateral biting tasks on right and left molars during both laboratory visits were plotted for each subject. Each plot comprised results from 5 static and 20 dynamic bites per side and visit and had a linear regression relationship determined. The 4 linear regression relationships for each subject were then averaged and from this, individual-specific mean masseter muscle activity for a $20 \mathrm{~N}$ bite force $(\mathrm{T} 20 \mathrm{~N}, \mu \mathrm{V})$ was calculated. The resulting T20N for each subject was used to establish magnitude thresholds to calibrate in-field ambulatory EMG recordings for quantification of masseter duty factors.

Masseter duty factor (DF): Duty factor, also known as duty time, ${ }^{22}$ is the percentage of time that a muscle is active, for defined thresholds, relative to the total duration of the recording ([Duration of muscle activity/Duration of recording period] x $100 \%$ ). The present study's focus was on low-magnitude masseter muscle activities (MMA), hence, DFs were calculated for the selected ranges of magnitude and duration thresholds of $\geq 5$ to $<25 \% \mathrm{~T} 20 \mathrm{~N}$ and $\geq 0.5$ to $<10$ s, respectively via a systematic process ${ }^{14}$ overall mean DFs were calculated that represented low-magnitude MMA for each subject during awake and sleep time-periods from the in-field EMG recordings. To prepare for DF calculations, each in-field ambulatory EMG recording was viewed, low-level (background) noise was filtered (multi-band gating, $-25 \mathrm{~dB}$ ) using commercial software (Wave Pad Sound Editor, Masters Edition, Out Browse LTD, Bialik, Israel) and any remaining signal noise was identified and excluded. Then task-definers were applied to calculate DF for the defined thresholds using customized software (MATLAB, The MathWorks, Inc., Natick, MA).

Categories of low-magnitude MMA: To differentiate between participants with relatively prolong and shorten low-grade masticatory muscle activity, a cutoff of 75th percentile was chosen, as it is a commonly reported statistic in the literature. For awake time-period, Group A consisted of individuals with overall DFs at or above 75th percentile whereas Group B contained residual participants. Similarly, for sleep time-period, Group Aconsistedof $\in \div$ alswithoverallDFsatorabove 75 thpercenti $\leq$ whereas GroupB contained remaining participants.

Presence of psychological symptoms: Each participant filled out self-reporting, reliable, and validated questionnaires: GAD$7,{ }^{18}$ PHQ- $9,{ }^{16}$ and PHQ-15, ${ }^{17}$ for the assessment of the severity of anxiety, depression, and somatic symptoms, respectively. Based on the responses and respective guidelines for the questionnaires, ${ }^{16-18}$ participants who were categorized as "Normal" or as having "Minimal "symptoms were combined into a single "Normal" group. Subjects characterized as Mild, Moderate, Moderate-Severe, and severe were combined into a single "Presence of symptoms" group.

No pain or pain-related TMD: Calibrated examiners with high levels of reliability, ranging from $K \geq 0.90-1$ for the clinical parameters conducted the DC-TMD examination. Subjects were categorized 
according to no pain, or pain-related TMD (myalgia, myofascial pain, and arthralgia), using the DC-TMD guidelines. ${ }^{20}$

Statistical analysis: A multi-step analytic approach was used to identify possible associations between the low-magnitude MMA and the presence of psychological symptoms and potential modifications of this association by the presence of pain-related TMD diagnoses and gender. The first step examined the distribution of the data relative to the 2 psychological groups (Normal, Presence of symptoms) using the Shapiro-Wilk test of normality. If the data showed a non-parametric distribution, logarithmic transformation was applied. The second step involved using independent t-tests to determine if there were statistical differences between the groups for age within the respective time-periods. In the third step, odds-ratios (OR) were determined for the association of the low-magnitude MMA and the presence of psychological symptoms. Similarly, the association between lowmagnitude MMA and psychological symptoms was stratified based on the pain-related TMD diagnoses and gender.

\section{Results}

The Shapiro-Wilk normality test indicated that the data were not normally distributed $(\mathrm{p}>.05)$; therefore, logarithmic transformation of the data was used. There were 68 participants in this pilot study, with a mean age of $33.1 \pm 12$ years, where $64.7 \%(\mathrm{~N}=44)$ were females (Table 1).

Table I Groups for time periods with gender and mean ages (standard deviation) of participants

\begin{tabular}{lllllll}
\hline & \multicolumn{1}{c}{ Total } & \multicolumn{5}{c}{ Males } \\
& Number & Mean age (yr.) & Number & Mean age (yr.) & Number & Mean age (yr.) \\
\hline Sample & 68 & $33.13(11.96)$ & 24 & $30.2(9.9)$ & 44 & $34.73(12.76)$ \\
Awake Time & & & & & & \\
Group A & 17 & $33.12(12.3)$ & 5 & $33.8(8.5)$ & 12 & $32.83(13.86)$ \\
Group B & 51 & $33.14(10.3)$ & 19 & $29.3(10.3)$ & 32 & $35.44(12.49)$ \\
Sleep Time & & & & & & \\
Group A & 17 & $31.06(9.70)$ & 9 & $26.2(5.7)$ & 8 & $36.50(10.68)$ \\
Group B & 51 & $33.82(12.64)$ & 15 & $32.6(11.3)$ & 36 & $34.33(13.28)$ \\
\hline
\end{tabular}

\section{Awake time-period}

Based on 75th percentile, participants were divided into Groups A (at or above 75th percentile) and B (all others), for the split analysis. For the awake period, in Group A, the mean age was 33.1 \pm 12.3 years and $70.6 \%$ were females $(\mathrm{n}=12)$ (Table 1$)$. No significant difference in age was detected between the Groups A and B in the awake timeperiod (independent t-test, pawake $=0.995$ ). Among the individuals reporting presence of anxiety symptoms, the odds ratio $(95 \%$ confidence intervals) of being in Group A was $1.6(0.5,4.8)$, however the increased odds of being in Group A versus Group B were not statistically significant for these individuals (Table 2). Among those reporting presence of depression and somatic symptoms, the odds ratios (95\% confidence intervals) of being in Group A were 3.3 (1.1, $10.3)$ and $4.3(1.2,15.0)$, respectively. These odds were found to be statistically significant ( $\mathrm{p}=0.036$ and 0.017 , respectively), indicating that individuals in Group A were more likely to report presence of depression and somatic symptoms compared to individuals in Group B (Table 2).

Table 2 Odds ratio (OR) analysis with $95 \%$ confidence intervals $(\mathrm{Cl})$ of association of low-magnitude masseter muscle activities (MMA) during awake timeperiod with self-reported anxiety, depression, and somatic symptoms and influence of pain-related TMD/no pain and gender on each respective association

\begin{tabular}{|c|c|c|c|c|c|c|c|c|c|c|c|c|c|c|c|c|}
\hline \multirow[b]{3}{*}{$\begin{array}{l}\text { Putative Risk } \\
\text { Factor }\end{array}$} & \multirow[b]{3}{*}{ Category } & \multicolumn{4}{|c|}{ Low-magnitude MMA } & \multirow[b]{3}{*}{ P value } & \multirow[b]{3}{*}{ OR } & \multirow{2}{*}{\multicolumn{5}{|c|}{$\begin{array}{c}\text { Pain-related TMD } \\
\text { Pain }(\mathrm{N}=\mathrm{I7}) \begin{array}{l}\text { No pain } \\
(\mathrm{N}=5 \mathrm{I})\end{array}\end{array}$}} & \multicolumn{4}{|c|}{ Gender } \\
\hline & & \multicolumn{2}{|c|}{$\begin{array}{l}\text { Group A } \\
(N=17)\end{array}$} & \multicolumn{2}{|c|}{$\begin{array}{l}\text { Group B } \\
(N=5 I)\end{array}$} & & & & & & & & Mal & le $(\mathrm{N}=24)$ & $\begin{array}{l}\text { Fem } \\
(\mathbf{N}=\end{array}$ & $\begin{array}{l}\text { nale } \\
\text { :44) }\end{array}$ \\
\hline & & $\mathbf{N}$ & $\begin{array}{l}\text { Column } \\
\%\end{array}$ & $\mathbf{N}$ & $\begin{array}{l}\text { Column } \\
\%\end{array}$ & & & $95 \% \mathrm{C}$ & IOR & $95 \% \mathrm{ClC}$ & \multicolumn{2}{|c|}{ R $95 \% \mathrm{Cl}$} & OR & $95 \% \mathrm{Cl}$ & \multicolumn{2}{|c|}{ OR $95 \% \mathrm{Cl}$} \\
\hline \multirow{2}{*}{ Anxiety symptoms } & Present & 9 & 52.9 & 21 & 41.2 & 0.398 & 1.6 & $0.5,4.8$ & 1.1 & $0.1,9.3$ & 1.6 & $0.4,6.0$ & 6.9 & $0.6,74.2$ & 0.9 & $0.2,3.6$ \\
\hline & Normal & 8 & 47.1 & 30 & 58.8 & & & & & & & & & & & \\
\hline \multirow{2}{*}{$\begin{array}{l}\text { Depression } \\
\text { symptoms }\end{array}$} & Present & 9 & 52.9 & 13 & 25.5 & $0.036 *$ & 3.3 & I.I, 10.3 & 35.3 & $0.6,46.0$ & 2.5 & $0.6,10.0$ & 15.0 & $1.3,174.4$ & 41.8 & $0.5,7.3$ \\
\hline & Normal & 8 & 47.1 & 38 & 74.5 & & & & & & & & & & & \\
\hline \multirow{2}{*}{ Somatic symptoms } & Present & 13 & 76.5 & 22 & 43.1 & $0.017 *$ & 4.3 & $1.2,15.0$ & 01.1 & $0.1,15.5$ & 5.5 & I.3, 24.4 & 3.3 & $0.4,24.8$ & 5.0 & $1.0,26.5$ \\
\hline & Normal & 4 & 23.5 & 29 & 56.9 & & & & & & & & & & & \\
\hline
\end{tabular}




\section{Sleep time-period}

ParticipantsweredividedintoGroupAandB, basedon 75 thpercentile In Group A, theme ana $\geq$ was $31.1 \pm 9.7$ yearsand $47.1 \%$ werefema $\leq \mathrm{s}(\mathrm{n}=8)$ $(\mathrm{Tab} \leq 1)$.Nosignificantd $\Leftrightarrow$ erence $\in \mathrm{a} \geq$ wasobservedbetween Groups $\mathrm{A}$ and $\mathrm{B}^{\prime}$ in the sleep time-period (independent t-test, $\mathrm{p}$ sleep=0.413).
Among the individuals reporting presence of anxiety, depression, and somatic symptoms, the odds ratio (95\% confidence intervals) of being in Group A were $0.9(0.3,2.6), 1.7(0.5,5.2)$, and $1.5(0.5$, $4.5)$, respectively. However, the increased odds were not statistically significant. Similarly, no statistically significant effect modifications were observed for pain-related TMD diagnoses or gender (Table 3).

Table 3 Odds ratio (OR) analysis with 95\% confidence intervals (CI) of association of low-magnitude masseter muscle activities (MMA) during sleep timeperiod with self-reported anxiety, depression, and somatic symptoms and influence of pain-related TMD/no pain and gender on each respective association

\begin{tabular}{|c|c|c|c|c|c|c|c|c|c|c|c|c|c|c|c|}
\hline & & \multicolumn{4}{|c|}{ Low-magnitude MMA } & & & & \multicolumn{4}{|c|}{ Pain-related TMD } & \multicolumn{2}{|c|}{ Gender } & \multirow[b]{2}{*}{$\begin{array}{l}\text { Female } \\
(N=44)\end{array}$} \\
\hline & & \multicolumn{2}{|c|}{$\begin{array}{l}\text { Group } A^{\prime} \\
(N=\mid 7)\end{array}$} & \multicolumn{2}{|c|}{$\begin{array}{l}\text { Group B' } \\
(\mathrm{N}=5 \mathrm{I})\end{array}$} & & & & \multicolumn{2}{|c|}{$\begin{array}{l}\text { Pain } \\
(N=\mid 7)\end{array}$} & \multicolumn{2}{|c|}{$\begin{array}{l}\text { No pain } \\
(N=5 I)\end{array}$} & \multicolumn{2}{|c|}{$\begin{array}{l}\text { Male } \\
(N=24)\end{array}$} & \\
\hline \multirow{3}{*}{$\begin{array}{l}\text { Putative Risk Factor } \\
\text { Anxiety symptoms }\end{array}$} & \multirow{2}{*}{$\begin{array}{l}\text { Category } \\
\text { Present }\end{array}$} & \multirow{2}{*}{$\begin{array}{l}\mathbf{N} \\
7\end{array}$} & \multirow{2}{*}{$\begin{array}{l}\begin{array}{l}\text { Column } \\
\%\end{array} \\
41.2\end{array}$} & \multirow{2}{*}{$\begin{array}{l}\mathbf{N} \\
23\end{array}$} & \multirow{2}{*}{$\begin{array}{l}\text { Column } \\
\% \\
45.1\end{array}$} & \multicolumn{2}{|c|}{ P valueOR } & $95 \% \mathrm{Cl}$ & IOR & \multicolumn{2}{|c|}{$95 \%$ CIOR } & \multirow{2}{*}{$\begin{array}{l}95 \% \mathrm{Cl} \\
0.4,4.9\end{array}$} & \multicolumn{2}{|c|}{ OR $95 \% \mathrm{Cl}$} & I OR $95 \% \mathrm{CI}$ \\
\hline & & & & & & 0.778 & 0.9 & $0.3,2.6$ & 0.2 & $0.01,2$. & 91.4 & & 1.9 & $0.4,10.0$ & $0.40 .1,2.1$ \\
\hline & Normal & 10 & 58.8 & 28 & 54.9 & & & & & & & & & & \\
\hline \multirow{2}{*}{$\begin{array}{l}\text { Depression } \\
\text { symptoms }\end{array}$} & Present & 7 & 42.1 & 15 & 29.4 & 0.369 & 1.7 & $0.5,5.2$ & - & - & 3.6 & $\mathrm{I}, \mathrm{I} 3.4$ & 2 & $0.3,11.5$ & $1.40 .3,7.0$ \\
\hline & Normal & 10 & 58.8 & 36 & 70.6 & & & & & & & & & & \\
\hline \multirow{2}{*}{ Somatic symptoms } & Present & & 58.8 & 25 & 49 & 0.484 & 1.5 & $0.5,4.5$ & 0.3 & $0.02,5$. & 62.5 & $0.7,8.6$ & 1.6 & $0.3,8.7$ & $2.40 .4,13.5$ \\
\hline & Normal & 7 & 41.2 & 26 & 51 & & & & & & & & & & \\
\hline
\end{tabular}

\section{Discussion}

The purpose of this pilot study was to determine if low-magnitude activities of the masseter muscle recorded in the individual's natural environments during awake and sleep periods were associated with self-reported anxiety, depression, and somatic symptoms. In addition, if associations existed, it was determined if these were modified by gender or by the presence of orofacial pain, such as pain-related TMD diagnoses. During the awake time-period, statistically significant associations were found between individuals who showed the highest duty factors for low-magnitude masseter muscle activities (Group A, at or above the 75th percentile of duty factors) and self-reported presence of depression and of somatic symptoms. These findings are similar to those reported by a similar investigation on the etiology of parafunctional activities, which result in marked masticatory muscle activation. ${ }^{23}$ The exact mechanism of this association is unknown. However, it has been suggested that presence of psychological distress may result in fear-avoidance behavior, ${ }^{21}$ which may further result in the individual holding her/his muscles taut or rigid, thus, causing increased muscle activity. Another proposed mechanism is the possible role of common neurotransmitters in the pathogenesis of psychological disorders and parafunctional activities. It has been suggested that disturbances in the central neurotransmitter system may account for psychological symptoms and parafunctional movements. $^{7}$ Presence of pain-related TMD strengthened the association between self-reported depression symptoms and Group A during awake periods. Similar associations have been reported in studies focused on oral movements resulting in pronounced muscle activations. ${ }^{23-25}$ A proposed model for the association between painrelated TMD and depression symptoms, suggests that these conditions may have similar pathogeneses. Neurotransmitters such as serotonin and norepinephrine have been implicated in both pain modulation and psychiatric disorders. Similarly, vulnerability to negative affectivity or dystrophic states may link psychological symptoms with somatic symptoms, including orofacial pain.

In contrast, absence of pain-related TMD augmented the association between self-reported somatic symptoms and Group A during the awake time-period. Previously, based on separate investigations, Mora et al. ${ }^{26}$ and Fernandes et al. ${ }^{27}$ both reported an association between jaw movements resulting in sleep period muscle activity (i.e. sleep bruxism) and the presence of pain-related TMD and somatic symptoms. It was suggested that this association was due to a shared pathophysiology associated with the dysregulation of hypothalamic-pituitary-adrenal stress hormone. However, in the present investigation no such association was observed between lowmagnitude masseter muscle activity, pain-related TMD, and somatic symptoms. A possible explanation for these contradictory findings can be attributed to the methodological differences between these investigations. In the study by Mora et al., ${ }^{27}$ muscle activities during sleep periods were evaluated by recording EMG burst activities. This is different from the present investigation where muscle activity was recorded irrespective of continuous or intermittent frequency of activity. Fernandes et al. ${ }^{27}$ used clinical examination and history for evaluation of nocturnal activity and focused on sleep bruxism only. In contrast, the present investigation used objective means to analyze low-magnitude masseter muscle activity during both functional and non-functional jaw movements in awake and sleep periods.

Male gender strengthened the odds of being in Group A and reporting the presence of depression symptoms during awake periods. While female gender augmented the odds of being in Group A and reporting the presence of somatic symptoms. Previous investigations have suggested statistically significant associations in females 
between presence of depression symptoms, somatic symptoms, and increased masticatory muscle activity. ${ }^{28,29}$ However, previous results like these should be interpreted with caution because the majority of these investigations have focused only on clinical cases. This can result in a sample bias where subjects have notably different physiological and psychological characteristics compared to those in the community. No association was found between the presence of psychological symptoms and low-magnitude MMA during sleep periods. These results are similar to the review findings of Manfredini \& Lobbezoo $^{7}$ who concluded that there was limited evidence in the literature supporting the association between nighttime parafunctional activity and presence of psychopathological symptoms. However, the results of the reviews were based on articles that had either used unreliable subjective clinical examinations or non-validated self-report questionnaires as a diagnostic criterion for parafunctional activity. ${ }^{7,30} \mathrm{An}$ important improvement in the current study was the use of calibrated ambulatory EMG to record muscle activity in participant's natural environment and use of validated questionnaires to assess psychological symptoms.

In the current study, the presence of anxiety symptoms was not associated with low-magnitude masseter muscle activity during awake and sleep periods. These findings contradict the stress-hyperactivity theory ${ }^{31}$ which asserts that hyperactivity is induced by psychological states, such as stress. To reiterate, the criteria used for diagnosis and quantification of masticatory muscle activity in previous investigations has not been consistent, and is characterized by potential bias and confounders, e.g. pain as a criterion for sleep bruxism. ${ }^{7,30}$ Additionally, the term "anxiety" which could indicate a temporary and nonpathological psychic state or a more complex psychiatric disorder, has frequently been used incorrectly. ${ }^{7,9}$ The current study has several limitations. First, this was a pilot study with relatively small and unbalanced sizes of some groups. Consequently, for some variables the widths of the confidence interval were very large. Because the width of the confidence interval for an individual odds ratio depends mainly on the sample size, these results suggest that future investigations be conducted with larger sample sizes with more balanced numbers in the sub-groups. Another possible limitation is cross-contamination of myoelectric signals from muscles other than the masseter, such as the buccinators, which is a nearby muscle of facial expression. This possibility exists because surface electrodes were used. ${ }^{32}$ On the other hand, surface electrodes have the advantages over fine-wire electrodes whereby "whole muscle" behavior is characterized rather than single motor units. In addition, use of fine-wire electrodes would have not been practical due to the considerable extent and nature of the in-field recording period (spanning over 2 time-periods for 3 days, each for a minimum of 6hours). Finally, only masseter muscle activity was used in the analysis. However, it has been shown previously that masseter and temporalis muscles are activated dissimilarly across different oral tasks. ${ }^{16}$ In the future, it would be important to see if there was an association of low-magnitude temporalis muscle activity with the presence of self-report of psychological symptoms during awake and sleep time-periods.

\section{Conclusion}

In this pilot study, participants with self-reported depression and somatic symptoms had significantly higher odds of having lowmagnitude masseter muscle activity during awake periods than those who did not report these symptoms. The association between low-magnitude MMA and self-reported depression symptoms was significantly augmented among male participants, whereas, the association between low-magnitude MMA and self-reported somatic symptoms were significantly strengthened among female participants without pain.

\section{Acknowledgement}

The authors wish to thank the study participants and Dr. Hongzeng Liu for customizing software programs used in data processing and analyses. This study was supported by NIH grant\# 5R01DE016417 -07 .

\section{Funding}

None.

\section{Conflicts of interest}

The authors declare no potential conflicts of interest with respect to the authorship and/ or publication of this article.

\section{References}

1. Farella M, Palla S, Erni S, et al. Masticatory muscle activity during deliberately performed oral tasks. Physiol Meas. 2008;29(12):1397-1410.

2. Uchida S, Iwasaki LR, Marx DB, et al. Variations in activities of human jaw muscles depend on tooth-tipping moments. Arch Oral Biol. 2008;53(2):199-205.

3. Gonzalez Y, Iwasaki LR, McCall WD, et al. Reliability of electromyographic activity vs. bite-force from human masticatory muscles. Eur J Oral Sci. 2011;119(3):219-224.

4. Pavone BW. Bruxism and its effect on the natural teeth. J Prosthet Dent 1985;53(5):692-696.

5. Ohrbach R, Fillingim RB, Mulkey F, et al. Clinical findings and pain symptoms as potential risk factors for chronic TMD: descriptive data and empirically identified domains from the OPPERA case-control study. $J$ Pain. 2011;12(11S):T27-T45.

6. Ohrbach R, Markiewicz MR, McCall WD, et al. Waking-state oral parafunctional behaviors: specificity and validity as assessed by electromyography. Eur J Oral Sci. 2008;116(5):438-444.

7. Manfredini D, Lobbezoo F. Role of psychosocial factors in the etiology of bruxism. J Orofac Pain. 2009;23(2):153-166.

8. Pierce CJ, Chrisman K, Bennett ME, et al. Stress, anticipatory stress, and psychologic measures related to sleep bruxism. J Orofac Pain. 1995;9(1):51-56.

9. Manfredini D, Fabbri A, Peretta R, et al. Influence of psychological symptoms on home-recorded sleep-time masticatory muscle activity in healthy subjects. J Oral Rehabil. 2011;38(12):902-911.

10. Manfredini D, Landi N, Fantoni F, et al. Anxiety symptoms in clinically diagnosed bruxers. J Oral Rehabil. 2005;32(8):584-588.

11. Glaros AG, Williams K. Tooth contact versus clenching: oral parafunctions and facial pain. J Orofac Pain. 2012;26(3):176-180.

12. Raphael KG, Janal MN, Sirois DA, et al. Masticatory muscle sleep background electromyographic activity is elevated in myofascial temporomandibular disorder patients. J Oral Rehabil. 2013;40(12):883891 .

13. Khawaja SN, McCall WD, Dunford R. In-field masticatory muscle activity in subjects with pain-related TMD diagnoses. Journal of Orthodontics and Craniofacial Research. 2014. 
14. Nasir, Shehryar KMS. Effects of Anxiety Symptoms on the Masticatory Muscle Activity of Subjects with TMD-Pain Diagnoses. ProQuest. 2014

15. Nickel JC, Gonzalez YM, McCall WD, et al. Muscle organization in individuals with and without pain and joint dysfunction. $J$ Dent Res. 2012;91(6):568-573.

16. Kroenke K, Spitzer RL, Williams JB. The PHQ-9: validity of a brief depression severity measure. J Gen Intern Med. 2001;16(9):606-613.

17. Kroenke K, Spitzer RL, Williams JB. The PHQ-15: validity of a new measure for evaluating the severity of somatic symptoms. Psychosom Med. 2002;64(2):258-266.

18. Spitzer RL, Kroenke K, Williams JB, et al. A brief measure for assessing generalized anxiety disorder: the GAD-7. Arch Intern Med. 2006;166(10):1092-1097.

19. Ohrbach R, Gonzalez Y, List T, et al. Diagnostic Criteria for Temporomandibular Disorders (DC/TMD) Clinical Examination Protocol. 2014;6:1-91

20. Schiffman E, Ohrbach R, Truelove E, et al. Diagnostic Criteria for Temporomandibular Disorders (DC/TMD) for Clinical and Research Applications: Recommendations of the International RDC/TMD Consortium Network* and Orofacial Pain Special Interest Groupdagger. J Oral Facial Pain Headache. 2014;28(1):6-27.

21. Leeuw M, Goossens ME, Linton SJ, et al. The fear-avoidance model of musculoskeletal pain: current state of scientific evidence. J Behav Med. 2007;30(1):77-94.

22. Grunheid T, Langenbach GE, Zentner A, et al. Duty time of rabbi jaw muscles varies with the number of activity bursts. $J$ Dent Res. 2006;85(12):1112-1117.

23. Bayar GR, Tutuncu R, Acikel C. Psychopathological profile of patients with different forms of bruxism. Clin Oral Investig. 2012;16(1):305-311.
24. Glaros AG, Williams K, Lausten L. The role of parafunctions, emotions and stress in predicting facial pain. J Am Dent Assoc. 2005;136(4):451458.

25. Michelotti A, Cioffi I, Festa P, et al. Oral parafunctions as risk factors for diagnostic TMD subgroups. J Oral Rehabil. 2010;37(3):157-162.

26. Mora SM, Weber D, Borkowski S, et al. Nocturnal masseter muscle activity is related to symptoms and somatization in temporomandibular disorders. J Psychosom Res. 2012;73(4):307-312.

27. Fernandes G, Franco AL, Siqueira JT, et al. Sleep bruxism increases the risk for painful temporomandibular disorder, depression and non-specific physical symptoms. J Oral Rehabil. 2012;39(7):538-544.

28. Winocur E, Littner D, Adams I, et al. Oral habits and their association with signs and symptoms of temporomandibular disorders in adolescents: a gender comparison. Oral Surg Oral Med Oral Pathol Oral Radiol Endod. 2006;102(4):482-487.

29. Kroenke K, Spitzer RL. Gender differences in the reporting of physical and somatoform symptoms. Psychosom Med. 1998;60(2):150-155.

30. Manfredini D, Lobbezoo F. Relationship between bruxism and temporomandibular disorders: a systematic review of literature from 1998 to 2008. Oral Surg Oral Med Oral Pathol Oral Radiol Endod. 2010;109(6):e26-e50.

31. Flor H, Turk DC. Psychophysiology of chronic pain: do chronic pain patients exhibit symptom-specific psychophysiological responses? Psychol Bull. 1989;105(2):215-259.

32. De Luca CJ, Gilmore LD, Kuznetsov M, et al. Filtering the surface EMG signal: Movement artifact and baseline noise contamination. J Biomech. 2010;43(8):1573-1579. 